\title{
A BILATERALLY DETERMINISTIC $\rho$-MIXING STATIONARY RANDOM SEQUENCE ${ }^{1}$
}

\author{
BY \\ RICHARD C. BRADLEY ${ }^{2}$
}

\begin{abstract}
A (nondegenerate) strictly stationary sequence $\left(X_{k}, k \in \mathbf{Z}\right)$ of random variables is constructed such that the $\rho$-mixing (maximal correlation mixing) condition is satisfied and each $X_{k}$ is measurable with respect to the double tail $\sigma$-field.
\end{abstract}

1. Introduction. Suppose $\left(X_{k}, k \in \mathbf{Z}\right)$ is a strictly stationary sequence of random variables on a probability space $(\Omega, \mathcal{F}, P)$. For $-\infty \leq J \leq L \leq \infty$ let $\mathcal{F}_{J}^{L}$ denote the $\sigma$-field of events generated by $\left(X_{k}, J \leq k \leq L\right)$. For the sequence $\left(X_{k}\right)$ the "past" and "future" tail $\sigma$-fields are respectively $\bigcap_{n=1}^{\infty} \mathcal{F}_{-\infty}^{-n}$ and $\bigcap_{n=1}^{\infty} \mathcal{F}_{n}^{\infty}$, and the "double" tail $\sigma$-field is $\bigcap_{n=1}^{\infty}\left(\mathcal{F}_{-\infty}^{-n} \vee \mathcal{F}_{n}^{\infty}\right)$. The sequence $\left(X_{k}\right)$ is said to be "bilaterally deterministic" if each of the r.v.'s $X_{k}$ is measurable with respect to the double tail $\sigma$-field.

Several authors have discussed examples of strictly stationary sequences $\left(X_{k}\right)$ which have a nontrivial double tail $\sigma$-field-or are even bilaterally deterministicsuch that the past and future tail $\sigma$-fields are each trivial. Olshen [5] gives an example with the $X_{k}$ 's in essence being real-valued. In Gurevic [2] and in Ornstein and Weiss [6], classes of finite-state examples are constructed, some of these examples being isomorphic to a Bernoulli shift. Also [6] refers to similar work of Furstenberg. Here we shall examine the question of what strong mixing conditions can be satisfied by bilaterally deterministic stationary sequences of real-valued r.v.'s. This work arose from a question posed to the author by $\mathrm{H}$. Berbee as to whether the (Rosenblatt) strong mixing condition implied a trivial double tail $\sigma$-field.

For any two $\sigma$-fields $A$ and $B$ define the "maximal correlation":

$$
\rho(\mathcal{A}, B):=\sup |\operatorname{Corr}(f, g)|, \quad f \in \mathcal{L}^{2}(\mathcal{A}), \quad g \in \mathcal{L}^{2}(B) .
$$

(In the notation $\mathcal{L}^{2}(\cdot)$ here, only real-valued random variables are included. Also, in the definition of correlation, $0 / 0$ is interpreted to be 0 .) For a given strictly stationary sequence $\left(X_{k}, k \in \mathbf{Z}\right)$, define for each $n \in \mathbf{N}, \rho(n):=\rho\left(\mathcal{F}_{-\infty}^{0}, \mathcal{F}_{n}^{\infty}\right)$. The sequence $\left(X_{k}\right)$ is said to be " $\rho$-mixing" if $\rho(n) \rightarrow 0$ as $n \rightarrow \infty$. The following theorem will be proved.

Received by the editors February 22, 1985 .

1980 Mathematics Subject Classification. Primary 60G10; Secondary 60G25.

Key words and phrases. Strictly stationary, strong mixing, $\rho$-mixing, double tail $\sigma$-field, bilaterally deterministic.

${ }^{1}$ This research was partially supported by NSF grant DMS 84-01021 and partially supported by AFOSR grant \#F49620 82 C 0009.

${ }^{2}$ Parts of this work were done while the author was a visitor at the Mathematical Centre in Amsterdam and the Center for Stochastic Processes in Chapel Hill. 
THEOREM 1. Suppose $c_{1}, c_{2}, c_{3}, \ldots$ is a sequence of positive numbers. Then there exists a bilaterally deterministic strictly stationary sequence $\left(X_{k}, k \in \mathbf{Z}\right)$ of nondegenerate real-valued random variables such that $\forall n \in \mathbf{N}, \rho(n) \leq c_{n}$.

In particular, a (nondegenerate) bilaterally deterministic strictly stationary sequence $\left(X_{k}\right)$ can be $\rho$-mixing with an arbitrarily fast rate of convergence of $\rho(n)$ to 0 . Now $\rho$-mixing implies the (Rosenblatt) strong mixing condition, which in turn implies that the past and future tail $\sigma$-fields are each trivial (see e.g. $[\mathbf{4}, \mathrm{pp}$. 301-306, Definition 17.1.1, Theorem 17.1.1, and Definition 17.2.1]).

It is well known that the "absolute regularity" condition [7] (also known under the name "weak Bernoulli") implies a trivial double tail $\sigma$-field. This fact is implicit in $[\mathbf{7}$, p. 194]. (To see why, observe from $[\mathbf{7},(4.1)]$ that absolute regularity implies $[\mathbf{7},(4.2)(\mathrm{a})]$, then use $[\mathbf{7}$, Lemma 4.3] to show that the double tail $\sigma$-field is trivial under the probability measure $\bar{P}$ (in [7]), and then combine these two facts to show it is also trivial under the given probability measure $P$.) As a consequence the $\varphi$-mixing condition, which is stronger than either absolute regularity or $\rho$-mixing and can be found in [4], also implies a trivial double tail $\sigma$-field.

The $\rho$-mixing condition plays a natural role in central limit theory for dependent r.v.'s (see e.g. Ibragimov [3]).

Theorem 1 is proved in $\S 3$. In $\S 2$ some preliminary work is done in preparation for that proof. Our construction in $\S \S 2$ and 3 is similar to (but more complicated than) Olshen's; his construction (see [5, p. 155, lines 5-10, and p. 156, lines 4-12]) contains some key ideas used in our construction.

2. Preliminaries. The following result of Csaki and Fischer [1, Theorem 6.2] will play a key role later on:

LEMMA 1 (CSAKI AND FISCHER). Suppose $A_{n}$ and $B_{n}, n \in \mathbf{N}$, are $\sigma$-fields, and the $\sigma$-fields $A_{n} \vee B_{n}, n \in \mathbf{N}$, are independent. Then

$$
\rho\left(\bigvee_{n \in \mathbf{N}} \mathcal{A}_{n}, \bigvee_{n \in \mathbf{N}} B_{n}\right)=\sup _{n \in \mathbf{N}} \rho\left(A_{n}, B_{n}\right) .
$$

For a proof see Witsenhausen $[\mathbf{8}$, Theorem 1]. (In that context there were only finitely many pairs of $\sigma$-fields, but the extension to Lemma 1 is elementary.)

Next, for any family $Z$ of random variables let $B(Z)$ denote the $\sigma$-field of events generated by $Z$.

LEMMA 2. Suppose $c_{1}, c_{2}, c_{3}, \ldots$ is a sequence of positive numbers. Then there exists a nondegenerate sequence $\left(Y_{k}, k \in \mathbf{Z}\right)$ of real-valued random variables such that the following two statements hold:

(i) $\forall n \in \mathbf{N}, \forall K \in \mathbf{Z}, \rho\left(B\left(Y_{k}, k \leq K\right), B\left(Y_{k}, k \geq K+n\right)\right) \leq c_{n}$.

(ii) $\forall K \in \mathbf{Z}, Y_{K}$ is measurable with respect to

$$
\bigcap_{n=1}^{\infty}\left(B\left(Y_{k}, k \leq-n\right) \vee B\left(Y_{k}, k \geq n\right)\right) .
$$

The sequence $\left(Y_{k}\right)$ that we shall construct for Lemma 2 will not be stationary, but it will obviously have the other properties needed for Theorem 1 . In $\S 3$ the stationary sequence $\left(X_{k}\right)$ for Theorem 1 will be constructed with the aid of a 
countable family of independent copies of the sequence $\left(Y_{k}\right)$ here. The rest of $\S 2$ is devoted to the proof of Lemma 2.

PROOF OF LEMMA 2. Without loss of generality we assume that

$$
1 \geq c_{1} \geq c_{2} \geq c_{3} \geq \cdots>0 .
$$

For each $n \in \mathbf{N}$ define

$$
r_{n}=c_{2 n} .
$$

(The purpose of (2.2) will become clear later on.)

On some probability space, let $\left(V_{k, m}: k \in \mathbf{Z}, m \in \mathbf{N}\right)$ be an array of random variables with the following three properties:

(2.3) For each $(k, m)$,

$$
P\left(V_{k, m}=-1\right)=P\left(V_{k, m}=1\right)=\frac{1}{2} .
$$

(2.4) The random variables $V_{0, m}, m \in \mathbf{N}$, and the random vectors $\left(V_{-n, m}, V_{n, m}\right)$, $n \in \mathbf{N}, m \in \mathbf{N}$, are all independent of each other.

(2.5) For each $n \in \mathbf{N}, m \in \mathbf{N}, i \in\{-1,1\}$ and $j \in\{-1,1\}$,

$$
P\left(V_{-n, m}=i, V_{n, m}=j\right)=\left(1+i j r_{n}\right) / 4 .
$$

Let $h: \mathbf{N} \rightarrow \mathbf{N}$ be a function such that for each $l \in \mathbf{N}$ the following two statements hold:

(2.6) There exist infinitely many odd integers $m \in \mathbf{N}$ such that $h(m)=l$.

(2.7) There exist infinitely many even integers $m \in \mathbf{N}$ such that $h(m)=l$.

For each $l \in \mathbf{N}, J \in \mathbf{N}$, define the two sets $S(l, J$,odd) and $S(l, J$, even) as follows: $S(l, J$, odd) (resp. $S(l, J$, even)) is the set of the least $J$ positive odd (resp. even) integers $m$ such that $h(m)=l$.

By (2.5) we have that $E V_{-n, m} V_{n, m}=r_{n}$ for each $n \in \mathbf{N}, m \in \mathbf{N}$; and by (2.4) and the strong law of large numbers, for each $n \in \mathbf{N}, l \in \mathbf{N}$,

$$
\operatorname{Lim}_{J \rightarrow \infty}\left(\frac{1}{J}\right) \cdot \sum_{m \in S(l, J, \text { odd })} V_{-n, m} V_{n, m}=r_{n} \quad \text { a.s. }
$$

and

$$
\operatorname{Lim}_{J \rightarrow \infty}\left(\frac{1}{J}\right) \cdot \sum_{m \in S(l, J, \text { even })} V_{-n, m} V_{n, m}=r_{n} \quad \text { a.s. }
$$

Deleting if necessary a null-set from the probability space on which the array $\left(V_{k, m}\right)$ is defined, we assume that for every sample point $\omega$ in this probability space the following two statements hold:

(2.8) $V_{k, m}(\omega) \in\{-1,1\}$ for every $k \in \mathbf{Z}, m \in \mathbf{N}$.

(2.9) For every $n \in \mathbf{N}, l \in \mathbf{N}$,

$$
\operatorname{Lim}_{J \rightarrow \infty}\left(\frac{1}{J}\right) \cdot \sum_{m \in S(l, J, \text { odd })} V_{-n, m}(\omega) \cdot V_{n, m}(\omega)=r_{n}
$$

and

$$
\operatorname{Lim}_{J \rightarrow \infty}\left(\frac{1}{J}\right) \cdot \sum_{m \in S(l, J, \text { even })} V_{-n, m}(\omega) \cdot V_{n, m}(\omega)=r_{n} .
$$


Let us digress for a moment to explain what will occur. Based on the array $\left(V_{k, m}\right)$, another array $\left(W_{k, m}\right)$ of $\{-1,1\}$-valued random variables will be defined, and for each $k \in \mathbf{Z}$ the r.v. $Y_{k}$ in Lemma 2 will simply be a one-to-one bimeasurable function of $\left(W_{k, 1}, W_{k, 2}, W_{k, 3}, \ldots\right)$. For each $n \in \mathbf{N}, l \in \mathbf{N}$, the r.v. $W_{-n, l}$ will be a function of the r.v.'s $\left(W_{-n-1, m}, W_{n+1, m}: m\right.$ odd, $\left.h(m)=l\right)$, and $W_{n, l}$ will be a function of $\left(W_{-n-1, m}, W_{n+1, m}: m\right.$ even, $\left.h(m)=l\right)$. In the end this will easily imply property (ii) in Lemma 2, but it has to be set up so that in the end property (i) of Lemma 2 will also hold.

Now let us define the array $\left(W_{k, m}: k \in \mathbf{Z}, m \in \mathbf{N}\right)$. First,

$$
\forall k \leq 0, \forall m \in \mathbf{N}, \quad W_{k, m}:=V_{k, m} .
$$

The definition of the other random variables $W_{k, m}$ will be recursive. For each $n \geq 0$, once $\left(W_{k, m}: k \leq n, m \in \mathbf{N}\right)$ is defined, define $W_{n+1, m}, m \in \mathbf{N}$, as follows:

$$
\begin{aligned}
& \forall \text { odd } m \in \mathbf{N}, \quad W_{n+1, m}:=W_{-n, h(m)} \cdot V_{n+1, m}, \\
& \forall \text { even } m \in \mathbf{N}, \quad W_{n+1, m}:=W_{n, h(m)} \cdot V_{n+1, m} .
\end{aligned}
$$

This completes the recursive definition.

In order to make our notation more compact, let us define for each $k \in \mathbf{Z}$ the random sequences $V_{k}$ and $W_{k}$ by $V_{k}:=\left(V_{k, 1}, V_{k, 2}, V_{k, 3}, \ldots\right)$ and $W_{k}:=\left(W_{k, 1}, W_{k, 2}\right.$, $\left.W_{k, 3}, \ldots\right)$. facts:

By (2.8), (2.10), (2.11) and an induction argument, we have the following two

(2.12) For every $k \in \mathbf{Z}, m \in \mathbf{N}$, the r.v. $W_{k, m}$ takes its values in $\{-1,1\}$ (at every sample point in our probability space).

(2.13) For every $n=0,1,2, \ldots$ the random sequences $W_{-n}$ and $W_{n}$ are measurable functions of $\left(V_{-n}, V_{-n+1}, \ldots, V_{n}\right)$.

Claim 1. For each $n \geq 0$ the random sequences $W_{-n}$ and $W_{n}$ are each a measurable function of $\left(W_{-n-1}, W_{n+1}\right)$.

Proof of Claim 1. Let $n \geq 0$ and $l \in \mathbf{N}$ be arbitrary but fixed. Then at every sample point in our probability space, by $(2.10),(2.11)$, the definition of $S(l, J$, odd $)$ and $(2.9)$,

$$
\begin{aligned}
\operatorname{Lim}_{J \rightarrow \infty} & \left(\frac{1}{J}\right) \cdot \sum_{m \in S(l, J, \text { odd })} W_{-n-1, m} W_{n+1, m} \\
& =\operatorname{Lim}_{J \rightarrow \infty}\left(\frac{1}{J}\right) \cdot \sum_{m \in S(l, J, \text { odd })} V_{-n-1, m} \cdot W_{-n, h(m)} V_{n+1, m} \\
& =W_{-n, l} \cdot \operatorname{Lim}_{J \rightarrow \infty}\left(\frac{1}{J}\right) \cdot \sum_{m \in S(l, J, \text { odd })} V_{-n-1, m} V_{n+1, m}=W_{-n, l} \cdot r_{n+1} .
\end{aligned}
$$

Hence $W_{-n, l}$ is a measurable function of $\left(W_{-n-1}, W_{n+1}\right)$. The same is true for $W_{n, l}$ by a similar argument using $S(l, J$, even). Claim 1 follows.

By Claim 1 and an induction argument we have the following:

Claim 2. For each $K \in \mathbf{Z}$, the random sequence $W_{K}$ is measurable with respect to $\bigcap_{n=1}^{\infty}\left(B\left(W_{k}, k \leq-n\right) \vee B\left(W_{k}, k \geq n\right)\right)$.

This will give us (ii) of Lemma 2 later on. Now we have to do some work to prepare for (i) of Lemma 2. 
Claim 3. Suppose $n \in \mathbf{N}, m \in \mathbf{N}$ and $Z$ is a random variable independent of $\left(V_{-n, m}, V_{n, m}\right)$ and taking its values in $\{-1,1\}$. Then the following two statements hold:

(i) The joint distribution of $\left(Z \cdot V_{-n, m}, Z \cdot V_{n, m}\right)$ is the same as that of $\left(V_{-n, m}\right.$, $\left.V_{n, m}\right)$.

(ii) $\rho\left(B\left(Z \cdot V_{-n, m}\right), B\left(Z \cdot V_{n, m}\right)\right)=\rho\left(B\left(V_{-n, m}\right), B\left(V_{n, m}\right)\right)=r_{n}$.

PROOF OF ClAIM 3. Part (i) follows from (2.5) and an elementary calculation. The first equality in (ii) follows from (i); we only need to verify the second equality in (ii).

Suppose $f$ (resp. $g$ ) is a nondegenerate function of $V_{-n, m}$ (resp. $V_{n, m}$ ). Since $V_{-n, m}$ takes only two values $(-1$ and +1$)$, it is easy to see that $f$ is automatically an affine function of $V_{-n, m}$. Similarly $g$ is an affine function of $V_{n, m}$. Hence $|\operatorname{Corr}(f, g)|=\left|\operatorname{Corr}\left(V_{-n, m}, V_{n, m}\right)\right|=r_{n}$ by (2.5) and a simple calculation. The second equation in (ii) follows, and Claim 3 is proved.

Claim 4. Suppose $n \geq 0$, and $Z_{1}, Z_{2}, Z_{3}, \ldots$ are $B\left(W_{k},-n \leq k \leq n\right)$-measurable random variables taking their values in $\{-1,1\}$. Then the $\sigma$-fields $B\left(W_{k},-n \leq k \leq\right.$ $n)$ and $B\left(Z_{J} \cdot V_{-n-1, J}, Z_{J} \cdot V_{n+1, J}\right), J=1,2,3, \ldots$, are all independent of each other.

Proof OF Claim 4. Let $J \geq 1$ be arbitrary but fixed. Define the $\sigma$-field

$$
A:=B\left(W_{k},-n \leq k \leq n\right) \vee\left(\bigvee_{h=1}^{J-1} B\left(Z_{h} \cdot V_{-n-1, h}, Z_{h} \cdot V_{n+1, h}\right)\right) .
$$

(In the case $J=1$ define $A:=B\left(W_{k},-n \leq k \leq n\right)$.) To prove Claim 4 it suffices to prove that the random vector $\left(Z_{J} \cdot V_{-n-1, J}, Z_{J} \cdot V_{n+1, J}\right)$ is independent of $A$.

Let $A \in A$ be an arbitrary fixed event such that $P(A)>0$. Let $i, j$ each be an arbitrary fixed element of $\{-1,1\}$. It suffices to prove that

$$
P\left(Z_{J} \cdot V_{-n-1, J}=i, Z_{J} \cdot V_{n+1, J}=j \mid A\right)=P\left(Z_{J} \cdot V_{-n-1, J}=i, Z_{J} \cdot V_{n+1, J}=j\right) .
$$

By (2.13) the random sequences $W_{-n}, W_{-n+1}, \ldots, W_{n}$ are (measurable) functions of $V_{-n}, V_{-n+1}, \ldots, V_{n}$. Consequently $A$ is an element of, and $Z_{J}$ is measurable with respect to, the $\sigma$-field $B\left(V_{k},-n \leq k \leq n\right) \vee B\left(V_{-n-1, h}, V_{n+1, h}: 1 \leq h \leq J-1\right)$; and $\left(V_{-n-1, J}, V_{n+1, J}\right)$ is independent of this $\sigma$-field by (2.4). Consequently, for each $z \in\{-1,1\}$ such that $P\left(A \cap\left\{Z_{J}=z\right\}\right)>0$, one has that

$$
\begin{aligned}
P\left(Z_{J} \cdot V_{-n-1, J}\right. & \left.=i, Z_{J} \cdot V_{n+1, J}=j \mid A \cap\left\{Z_{J}=z\right\}\right) \\
& =P\left(V_{-n-1, J}=i / z, V_{n+1, J}=j / z \mid A \cap\left\{Z_{J}=z\right\}\right) \\
& =P\left(V_{-n-1, J}=i / z, V_{n+1, J}=j / z\right) \\
& =\left(1+r_{n+1} i j / z^{2}\right) / 4=\left(1+r_{n+1} i j\right) / 4 \\
& =P\left(Z_{J} \cdot V_{-n-1, J}=i, Z_{J} \cdot V_{n+1, J}=j\right) .
\end{aligned}
$$

(We are using (2.5) and Claim 3(i).) Equation (2.14) now follows by a simple argument, and this completes the proof of Claim 4.

In applying Claim 4 in what follows, we shall sometimes implicitly take $Z_{J}:=1$, in which case $B\left(Z_{J} V_{-n-1, J}, Z_{J} V_{n+1, J}\right)=B\left(V_{-n-1, J}, V_{n+1, J}\right)$. 
Claim 5. For each $n \in \mathbf{N}$ the following two statements hold:

(i) $\rho\left(\mathrm{B}\left(W_{k},-n \leq k \leq n-1\right), \mathrm{B}\left(W_{n}\right)\right) \leq r_{n}$.

(ii) $\rho\left(B\left(W_{-n}\right), B\left(W_{k},-n+1 \leq k \leq n\right)\right) \leq r_{n}$.

PROOF OF ClaIM 5. Let $n \in \mathbf{N}$ be arbitrary but fixed.

ProOF OF Claim 5(i). Define the $\sigma$-fields $\mathcal{A}_{j}$ and $B_{j}, j=0,1,2, \ldots$, as follows: $A_{0}:=B\left(W_{k},-n+1 \leq k \leq n-1\right)$ and $B_{0}:=\{\Omega, \phi\}$. For odd $j \geq 1, A_{j}:=$ $B\left(W_{-n+1, h(j)} \cdot V_{-n, j}\right)$ and $B_{j}:=B\left(W_{-n+1, h(j)} \cdot V_{n, j}\right)$. For even $j \geq 2, A_{j}:=$ $\mathrm{B}\left(W_{n-1, h(j)} \cdot V_{-n, j}\right)$ and $\mathrm{B}_{j}:=\mathrm{B}\left(W_{n-1, h(j)} \cdot V_{n, j}\right)$. (Here of course $\Omega$ denotes the sample space.) Define the $\sigma$-fields $A$ and $B$ by

$$
A:=\bigvee_{j=0}^{\infty} A_{j} \text { and } B:=\bigvee_{j=0}^{\infty} B_{j} \text {. }
$$

For each odd $j \in \mathbf{N}$, by (2.10) and (2.12),

$$
W_{-n, j}=V_{-n, j}=\left(W_{-n+1, h(j)} \cdot V_{-n, j}\right) \cdot W_{-n+1, h(j)},
$$

and since $\left(W_{-n+1, h(j)} \cdot V_{-n, j}\right)$ and $W_{-n+1, h(j)}$ are each $A$-measurable, $W_{-n, j}$ is $\mathcal{A}$-measurable. Similarly, for each even $j \in \mathbf{N}, W_{-n, j}$ is $A$-measurable. Hence $W_{-n}$ is $A$-measurable. (Of course $W_{-n+1}, \ldots, W_{n-1}$ are also $A$-measurable.)

Also, for each $j \geq 1, B_{j}=B\left(W_{n, j}\right)$ by $(2.11)$; and hence $B=B\left(W_{n}\right)$. Hence

$$
\rho\left(B\left(W_{-n}, W_{-n+1}, \ldots, W_{n-1}\right), B\left(W_{n}\right)\right) \leq \rho(\mathcal{A}, B)=\sup _{j \geq 0} \rho\left(\mathcal{A}_{j}, B_{j}\right)=r_{n} .
$$

Here the inequality is trivial, the first equality holds by Claim 4 and Lemma 1, and the second equality holds by Claim 3 . This completes the proof of (i).

PROOF OF ClaIM 5(ii). This is analogous to the proof of (i), but with a few changes. One defines the $\sigma$-fields $A_{j}$ and $B_{j}$ as follows:

$$
A_{0}:=\{\Omega, \phi\} \quad \text { and } \quad B_{0}:=B\left(W_{-n+1}, \ldots, W_{n-1}\right) .
$$

For all $j \geq 1, A_{j}:=B\left(V_{-n, j}\right)$ and $B_{j}:=B\left(V_{n, j}\right)$. Again one defines $A:=\bigvee_{j=0}^{\infty} A_{j}$ and $B:=\bigvee_{j=0}^{\infty} B_{j}$. Then one observes that $A=B\left(W_{-n}\right)$ and that $W_{n}$ (as well as $\left.W_{-n+1}, \ldots, W_{n-1}\right)$ is $B$-measurable. The proof of (ii) is then completed in the same way as (i).

Claim 6. For each $n \in \mathbf{N}$ the following two statements hold:

(i) $\rho\left(B\left(W_{k}, k \leq n-1\right), B\left(W_{k}, k \geq n\right)\right) \leq r_{n}$.

(ii) $\rho\left(B\left(W_{k}, k \leq-n\right), B\left(W_{k}, k \geq-n+1\right)\right) \leq r_{n}$.

PROOF OF Claim 6. Let $n \in \mathbf{N}$ be arbitrary but fixed.

ProOF OF ClAIM 6(i). It suffices to prove that $\forall N \geq n$,

$$
\rho\left(B\left(W_{k},-N \leq k \leq n-1\right), B\left(W_{k}, n \leq k \leq N\right)\right) \leq r_{n} .
$$

We shall show this by induction on $N$. By Claim 5 ,

$$
\rho\left(B\left(W_{k},-n \leq k \leq n-1\right), B\left(W_{n}\right)\right) \leq r_{n} .
$$

Now we only need to carry out the induction step.

Assume that $N \geq n$ is such that

$$
\rho\left(B\left(W_{k},-N \leq k \leq n-1\right), B\left(W_{k}, n \leq k \leq N\right)\right) \leq r_{n} .
$$

To prove Claim 6(i) it suffices to show that

$$
\rho\left(B\left(W_{k},-N-1 \leq k \leq n-1\right), B\left(W_{k}, n \leq k \leq N+1\right)\right) \leq r_{n} .
$$


The proof will be somewhat similar to that of Claim 5 .

Define the $\sigma$-fields $\mathcal{A}_{j}$ and $B_{j}, j=0,1,2, \ldots$, as follows:

$$
A_{0}:=B\left(W_{k},-N \leq k \leq n-1\right) \quad \text { and } \quad B_{0}:=B\left(W_{k}, n \leq k \leq N\right) \text {. }
$$

For each odd $j \geq 1, A_{j}:=B\left(W_{-N, h(j)} \cdot V_{-N-1, j}\right)$ and $B_{j}:=B\left(W_{-N, h(j)} \cdot V_{N+1, j}\right)$. For each even $j \geq 2, A_{j}:=B\left(V_{-N-1, j}\right)$ and $B_{j}:=B\left(V_{N+1, j}\right)$. Define the $\sigma$-fields $A$ and $B$ by

$$
A:=\bigvee_{j=0}^{\infty} A_{j} \text { and } B:=\bigvee_{j=0}^{\infty} B_{j} .
$$

For each odd $j \in \mathbf{N}$, by (2.10) and (2.12), $W_{-N-1, j}=V_{-N-1, j}=\left(W_{-N, h(j)}\right.$. $\left.V_{-N-1, j}\right) \cdot W_{-N, h(j)}$, which is a product of two $A$-measurable r.v.'s, and hence $W_{-N-1, j}$ is $A$-measurable. For each even $j \in \mathbf{N}, W_{-N-1, j}=V_{-N-1, j}$, which is $A$-measurable. Hence $W_{-N-1}$ is $A$-measurable.

For each odd $j \in \mathbf{N}$, by (2.11), $W_{N+1, j}=W_{-N, h(j)} \cdot V_{N+1, j}$, which is Bmeasurable. For each even $j \in \mathbf{N}, W_{N+1, j}=W_{N, h(j)} \cdot V_{N+1, j}$, which is a product of $B$-measurable functions and is therefore $B$-measurable. Hence $W_{N+1}$ is $B$ measurable.

Consequently,

$$
\begin{aligned}
\rho\left(B \left(W_{k},-N-1\right.\right. & \left.\leq k \leq n-1), B\left(W_{k}, n \leq k \leq N+1\right)\right) \\
& \leq \rho(\mathcal{A}, B)=\sup _{j \geq 0} \rho\left(A_{j}, B_{j}\right) \leq \max \left\{r_{n}, r_{N+1}\right\}=r_{n} .
\end{aligned}
$$

Here the first inequality is trivial, we are using Claim 4 and Lemma 1 to obtain the first equality, and then Claim 3 and the induction hypothesis to obtain the second inequality, and finally (2.1) and (2.2) to obtain the second equality. This completes the induction step, and Claim 6(i) is proved.

ProOF OF ClaIM 6(ii). This is essentially identical to the proof of (i). One has to change the definition of $A_{0}$ and $B_{0}$ to $A_{0}:=B\left(W_{k},-N \leq k \leq-n\right)$ and $B_{0}:=B\left(W_{k},-n+1 \leq k \leq N\right)$.

Claim 7. If $n \in \mathbf{N}$ and $K \in \mathbf{Z}$, then $\rho\left(B\left(W_{k}, k \leq K\right), B\left(W_{k}, k \geq K+n\right)\right) \leq c_{n}$.

PROOF OF ClaIM 7. Let us first consider the case where $n$ is odd, say $n=$ $2 m-1$ where $m \in \mathbf{N}$. Then either $K \leq-m$ or $K+n \geq m$. If $K \leq-m$, then $-2 K \geq 2 m>n$, and

$$
\begin{aligned}
\rho\left(B \left(W_{k}, k\right.\right. & \left.\leq K), B\left(W_{k}, k \geq K+n\right)\right) \\
& \leq \rho\left(B\left(W_{k}, k \leq K\right), B\left(W_{k}, k \geq K+1\right)\right) \leq r_{-K}=c_{-2 K} \leq c_{n}
\end{aligned}
$$

by Claim 6(ii), (2.2) and (2.1). If instead $K+n \geq m$, then a similar argument (using Claim 6(i)) works. The proof for even $n$ is similar to that for odd $n$.

The rest of the proof of Lemma 2 is now a trivial, cosmetic formality. Let $f:\{-1,1\} \times\{-1,1\} \times\{-1,1\} \times \cdots \rightarrow \mathbf{R}$ be a bimeasurable isomorphism. (It is well known that such an $f$ exists.) Define the sequence $\left(Y_{k}, k \in \mathbf{Z}\right)$ by $Y_{k}:=$ $f\left(W_{k}\right) \forall k \in \mathbf{Z}$. Properties (i) and (ii) of Lemma 2 now follow immediately from Claims 7 and 2 respectively. This completes the proof of Lemma 2.

3. Proof of Theorem 1. Let $c_{1}, c_{2}, c_{3}, \ldots$ be as in the statement of Theorem 1. Our first step is to construct countably many copies of the probability space and random sequence $\left(Y_{k}, k \in \mathbf{Z}\right)$ from Lemma 2 . Then on the product probability 
space we obtain an array $\left(Y_{k}^{(j)}: k \in \mathbf{Z}, j \in \mathbf{Z}\right)$ of real-valued random variables with the following three properties:

(3.1) The sequences $\left(Y_{k}^{(j)}, k \in \mathbf{Z}\right), j=\ldots,-1,0,1, \ldots$, are nondegenerate and are independent of each other, and they have the same distribution on $\cdots \times \mathbf{R} \times$ $\mathbf{R} \times \mathbf{R} \times \cdots$.

(3.2) For each $j \in \mathbf{Z}, n \in \mathbf{N}$ and $K \in \mathbf{Z}, \rho\left(B\left(Y_{k}^{(j)}, k \leq K\right), B\left(Y_{k}^{(j)}, k \geq K+n\right)\right) \leq$ $c_{n}$.

(3.3) For each $j \in \mathbf{Z}$ and $K \in \mathbf{Z}$, the r.v. $Y_{K}^{(j)}$ is measurable with respect to

$$
\bigcap_{n=1}^{\infty}\left(B\left(Y_{k}^{(j)}, k \leq-n\right) \vee B\left(Y_{k}^{(j)}, k \geq n\right)\right) \text {. }
$$

Let $g: \cdots \times \mathbf{R} \times \mathbf{R} \times \mathbf{R} \times \cdots \rightarrow \mathbf{R}$ be a bimeasurable isomorphism. (It is well known that such a function $g$ exists.) Define the sequence $\left(X_{k}, k \in \mathbf{Z}\right)$ of real-valued random variables as follows:

$$
\forall k \in \mathbf{Z} \quad X_{k}:=g\left(\ldots, Y_{-1}^{(k-1)}, Y_{0}^{(k)}, Y_{1}^{(k+1)}, \ldots\right) .
$$

By a simple argument using (3.1), this sequence $\left(X_{k}\right)$ is nondegenerate and strictly stationary.

For each $n \in \mathbf{N}$, by (3.4),

$$
B\left(X_{k}, k \leq-n\right) \vee B\left(X_{k}, k \geq n\right)=\bigvee_{j \in \mathbf{Z}}\left(B\left(Y_{k}^{(j)}, k \geq j+n\right) \vee B\left(Y_{k}^{(j)}, k \leq j-n\right)\right) \text {. }
$$

Hence for each $n \in \mathbf{N}$ and $K \in \mathbf{Z}$, the r.v. $X_{K}$ is measurable with respect to $B\left(X_{k}, k \leq-n\right) \vee B\left(X_{k}, k \geq n\right)$, by a simple argument using (3.3) and (3.4). Hence the sequence $\left(X_{k}\right)$ is bilaterally deterministic.

For each $n \in \mathbf{N}$,

$$
\begin{aligned}
\rho(n) & :=\rho\left(B\left(X_{k}, k \leq 0\right), B\left(X_{k}, k \geq n\right)\right) \\
& =\rho\left(\bigvee_{j \in \mathbf{Z}} B\left(Y_{k}^{(j)}, k \geq j\right), \bigvee_{j \in \mathbf{Z}} B\left(Y_{k}^{(j)}, k \leq j-n\right)\right) \\
& =\sup _{j \in \mathbf{Z}} \rho\left(B\left(Y_{k}^{(j)}, k \geq j\right), B\left(Y_{k}^{(j)}, k \leq j-n\right)\right) \leq c_{n}
\end{aligned}
$$

by (3.4), (3.1), Lemma 1 and (3.2). This completes the proof of Theorem 1.

ACKNOWLEDGMEnTs. The author is indebted to Henry Berbee for suggesting this topic, and for calling attention to the earlier references. For their hospitality during which parts of this work were completed, the author also thanks Włodzimierz and Grazyna Bryc as well as the Mathematical Centre in Amsterdam and the Center for Stochastic Processes in Chapel Hill.

\section{REFERENCES}

1. P. Csaki and J. Fischer, On the general notion of maximal correlation, Magyar Tud. Akad. Mat. Kutató Int. Közl. 8 (1963), 27-51.

2. V. M. Gurevic, On one-sided and two-sided regularity of stationary random processes, Soviet Math. Dokl. 14 (1973), 804-808.

3. I. A. Ibragimov, $A$ note on the central limit theorem for dependent random variables, Theory Probab. Appl. 20 (1975), 135-141. 
4. I. A. Ibragimov and Y. V. Linnik, Independent and stationary sequences of random variables, Wolters-Noordhoff, Groningen, 1971.

5. R. A. Olshen, The coincidence of measure algebras under an exchangable probability, $\mathrm{Z}$. Wahrsch. Verw. Gebiete 18 (1971), 153-158.

6. D. S. Ornstein and B. Weiss, Every transformation is bilaterally deterministic, Israel J. Math. 21 (1975), 154-158.

7. V. A. Volkonskii and Y. A. Rozanov, Some limit theorems for random functions. II, Theory Probab. Appl. 6 (1961), 186-198.

8. H. S. Witsenhausen, On sequences of pairs of dependent random variables, SIAM J. Appl. Math. 28 (1975), 100-113.

Department of Mathematics, Indiana University, Bloomington, Indiana 47405 